\title{
Manejo analgésico postoperatorio con bloqueos regionales guiados por ultrasonografía en cirugía de hombro
}

\section{Postoperative analgesic management with ultrasonography guided regional blocks in shoulder surgery}

\author{
Yazmín Galván Talamantes, ${ }^{*}$ Gerardo Esteban Álvarez Reséndiz, ${ }^{*}$ Juventino Cisneros García, ${ }^{\ddagger}$ \\ Mercedes Nicté López Hernández,§ José Rodrigo Fernández Soto,§ Mario Fajardo Pérez"
}

\section{RESUMEN}

El dolor postoperatorio en la actualidad es una condición no manejada adecuadamente. En las últimas dos décadas diversos estudios han demostrado que $70 \%$ o más de los pacientes quirúrgicos reportaron dolor moderado a severo después de la cirugía. En el manejo preoperatorio y postoperatorio deben considerarse técnicas de analgesia multimodal con la incorporación de anestesia regional. En el manejo exitoso del dolor postoperatorio en la cirugía de hombro, la anestesia regional con el uso de ultrasonografía provee múltiples ventajas entre las que destacan menor incidencia de complicaciones, un egreso hospitalario anticipado, mejores condiciones del paciente para la rehabilitación y disminución de un reingreso no previsto, con el consecuente decremento en los costos de hospitalización. Actualmente los bloqueos interfasciales se han incluido en las técnicas tradicionales de anestesia regional, destacando el bloqueo de la fascia del erector espinal (ESP) para las cirugías de hombro. Objetivo: Describir la importancia de los bloqueos regionales con ultrasonografía para manejo del dolor postoperatorio en cirugía de hombro haciendo énfasis en la incorporación del bloqueo ESP.

Palabras clave: Analgesia, cirugía de hombro, bloqueos regionales, bloqueo erector de la espina, ultrasonido.

Nivel de evidencia: III

\begin{abstract}
Postoperative pain is currently a condition not adequately managed, in the last two decades various studies have shown that $70 \%$ or more of surgical patients reported moderate to severe pain after surgery; both preoperative and postoperative management of the patient should be considered, techniques of multimodal analgesia and regional anesthesia. In the successful management of postoperative pain in shoulder surgery, regional anesthesia with the use of ultrasound provides multiple advantages, among which are the lower incidence of complications, an early hospital discharge, better patient conditions for rehabilitation, and less re-admission, with the consequent decrease in hospitalization costs; At present, interfascial blocks have been included in the traditional techniques highlighting for shoulder surgeries the spinal erector fascia block (ESP). Objective: To highlight the importance of regional blockages with ultrasonography for postoperative anesthetic management in shoulder surgery, emphasizing the incorporation of the erector muscle block of the spine.
\end{abstract}

Keywords: Analgesia, shoulder surgery, regional blockages, spinal erector block, ultrasound.

Level of evidence: III
* Departamento de Anestesiología, Centro Médico ABC Campus Santa Fe, The American British Cowdray Medical Center.

₹ Departamento de Traumatología y Ortopedia, Centro Médico ABC Campus Santa Fe, The American British Cowdray Medical Center.

$\S$ Residente de Anestesiología del Hospital Ángeles Lomas.

" Anestesiólogo del Hospital Universitario de Móstoles, Madrid, España.
Recibido para publicación: 15/03/2020. Aceptado: 15/06/2020.

Correspondencia:

Dra. Yazmín Galván Talamantes

Departamento de Anestesiología Centro Médico ABC.

E-mail: yazmingalvan21@gmail.com 


\section{INTRODUCCIÓN}

De acuerdo con antiguos reportes en jeroglíficos egipcios de 3,000 años de antigüedad la cirugía de hombro se practicaba con técnicas de apalancamiento para la reducción de hombro. ${ }^{1}$ En la actualidad los avances tecnológicos y la comprensión de la anatomía funcional han generado el desarrollo de la cirugía artroscópica de hombro, la artrografía de hombro en el año de $1965^{2}$ es un antecedente importante que precedió a la artroscopia de hombro, la cual representa uno de los mayores avances ortopédicos del siglo pasado, la misma técnica se ha perfeccionado aplicándose a una amplia variedad de procedimientos ortopédicos y múltiples patologías de hombro, la cual representa la cirugía de hombro más frecuentemente realizada; ${ }^{1}$ sin embargo, el manejo del dolor postoperatorio severo sigue siendo un importante desafío para muchos anestesiólogos. ${ }^{3}$ Existe una amplia variedad de pacientes que se presentan a este tipo de intervenciones, incluyendo pacientes atletas, mesomórficos, jóvenes con lesiones deportivas que requieren un procedimiento de estabilización; igualmente es común el paciente reumatoide, geriátrico, frágil que requiere descompresión articular o artroplastia. El manejo de estos pacientes requiere una evaluación y preparación preoperatoria íntegra, un manejo intraoperatorio cuidadoso y el uso apropiado de técnicas anestésicas regionales que proporcionen un alivio adecuado del dolor en el periodo postoperatorio inmediato y en las primeras 48 horas. Las técnicas de anestesia regional tienen la capacidad de controlar el dolor de manera efectiva tanto en reposo como en movimiento, reducen los espasmos musculares, permiten una movilización e incorporación más tempranas a fisioterapia, menor estancia intrahospitalaria y mayor satisfacción del paciente. ${ }^{4-6}$ Por lo tanto, estas técnicas regionales tienen el potencial de mejorar tanto la recuperación y rehabilitación del paciente como el resultado después de cirugías abiertas y artroscópicas.

\section{Anatomía e inervación del hombro}

El hombro está compuesto por una interacción dinámica de huesos, articulaciones, músculos y ligamentos, se considera la articulación más móvil del cuerpo humano. Lo integran tres huesos: (húmero, escápula y clavícula) y dos articulaciones: la articulación acromioclavicular y glenohumeral. Los músculos y ligamentos del hombro permiten y restringen el movimiento, igualmente participan en la es- tabilización activa y pasiva. ${ }^{7}$ La estabilidad estática del hombro es proporcionada por el lábrum, cápsula y ligamento glenohumeral, mientras que el mango rotador, la cabeza larga del tendón del bíceps y los músculos periescapulares proporcionan estabilidad dinámica. ${ }^{8}$ El plexo braquial provee todas las funciones motoras y sensoriales del hombro, excepto las cutáneas cefálicas, que están inervadas por los nervios supraclaviculares que se originan del plexo cervical superficial (C4-C3). Para un adecuado control eficaz del dolor postoperatorio del hombro se requiere inhibir el suministro nervioso a la membrana sinovial, cápsula, superficies articulares, periostio, ligamentos y los músculos del hombro. Las ramas terminales del plexo braquial que otorgan inervación a la mayoría del hombro son los nervios supraescapular y axilar; el primero suministra inervación sensorial subacromial a la articulación acro-

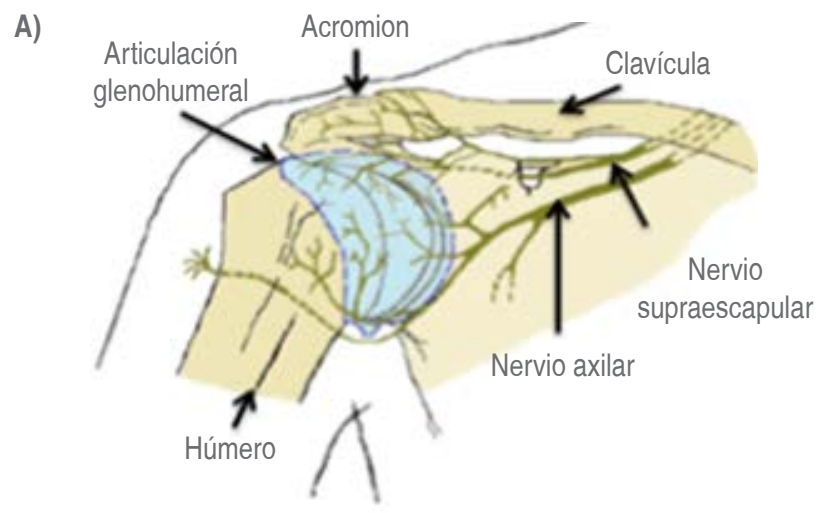

B)

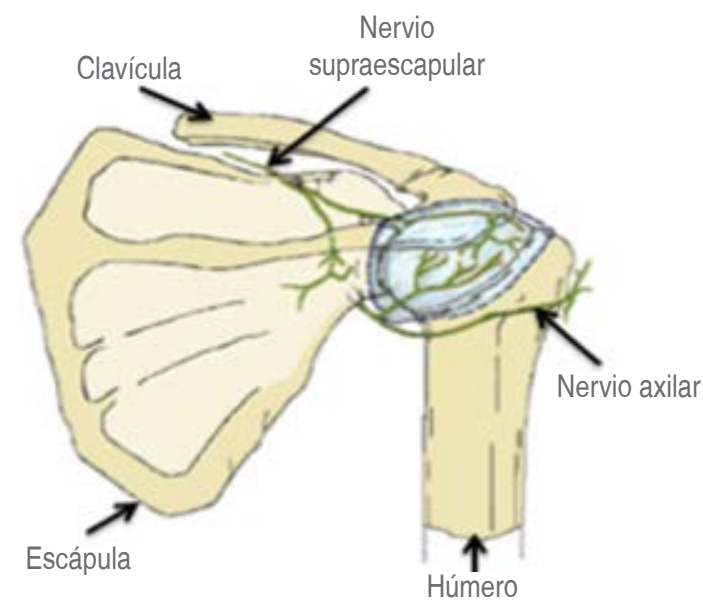

Figura 1: A) Inervación anterior de la articulación del hombro. El nervio supraescapular y el nervio axilar son los nervios primarios que inervan la cápsula y la articulación glenohumeral. B) Inervación posterior de la articulación del hombro. Ambos nervios, supraescapular y axilar, también proporcionan inervación a la cápsula posterior del hombro. 
An Med (Mex) 2020; 65 (2): 114-121

mioclavicular y ligamento coracoclavicular, $70 \%$ de la cápsula de la articulación del hombro surge del tronco superior del plexo (C5-C6), también proporciona inervación a la cápsula posterior del hombro, articulación acromioclavicular, bolsa subacromial y ligamento coracoclavicular ${ }^{9}$ (Figura 1 A y B). El nervio axilar se origina de las raíces nerviosas de (C5-C6) derivándose del cordón posterior del plexo braquial, el nervio axilar penetra en la cara anteroinferior del músculo subescapular donde cruza dividiéndose en dos troncos, el tronco anterior suministra la inervación motora al músculo deltoides anterior y medio, el tronco posterior emite una rama al músculo redondo menor y al deltoides posterior antes de terminar como el nervio cutáneo braquial lateral superior que suministra la inervación cutánea de la piel que cubre el deltoides. ${ }^{10}$

\section{Bloqueo interescalénico}

El bloqueo interescalénico es el estándar de oro para la analgesia del hombro y el bloqueo más utilizado para los procedimientos de hombro; fue descrito por el Dr. Winnie en $1970 .{ }^{11}$ Se bloquean las raíces nerviosas de C5, C6 en el tronco superior, dependiendo del volumen del anestésico local utilizado, serán las raíces nerviosas de C7 y C8 las que podrán alcanzarse. El bloqueo es especialmente útil para procedimientos que involucren al hombro, incluidos los dos tercios laterales de la clavícula, el húmero proximal y la articulación del hombro. El bloqueo puede realizarse con una inyección única o un bloqueo continuo; en la actualidad se puede eje-

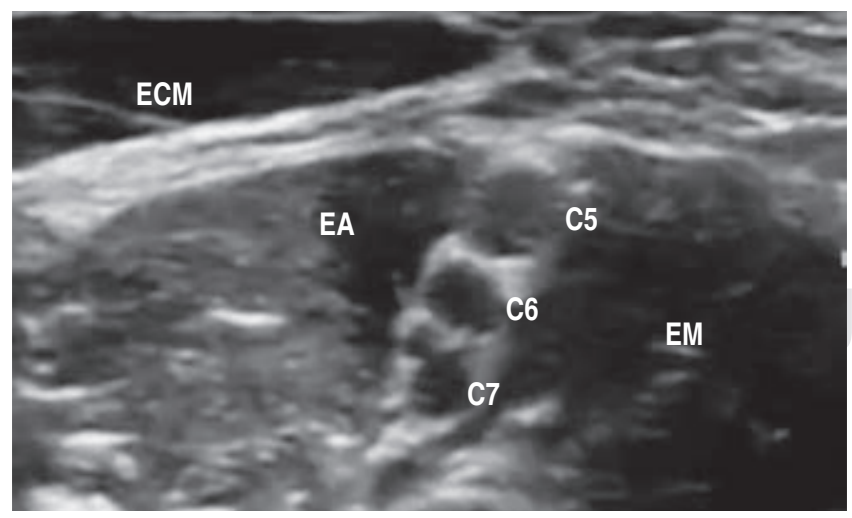

Figura 2: Identificación por ultrasonido del plexo braquial entre los músculos escaleno medio y escaleno anterior por debajo del músculo esternocleidomastoideo. Visualizándose a una profundidad de $1-3 \mathrm{~cm}$.

$\mathrm{EM}=$ músculo escaleno medio. $\mathrm{EA}=$ músculo escaleno anterior. $\mathrm{ECM}=$ músculo esternocleidomastoideo.

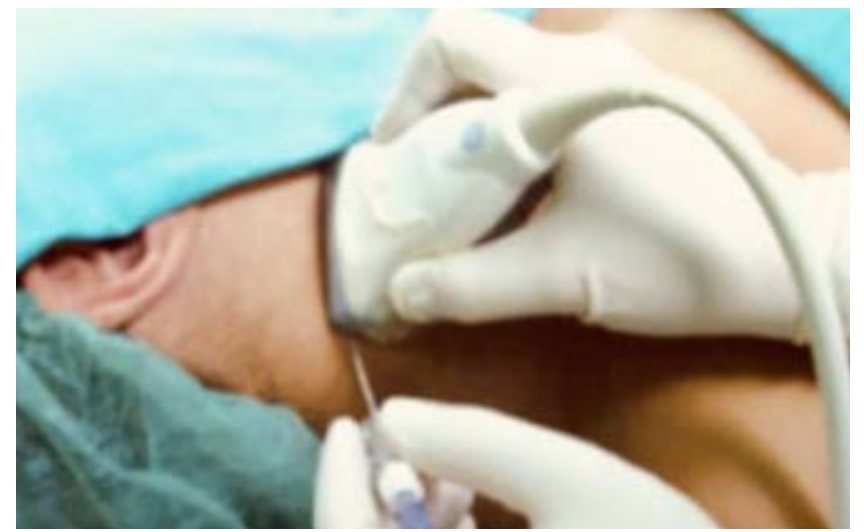

Figura 3: Técnica en plano. Sonda lineal en posición transversal, de 3 a 4 $\mathrm{cm}$ por arriba de la clavícula a nivel del cartílago cricoides.

cutar con la técnica de estimulación nerviosa guiada por ultrasonido o ambas. ${ }^{12,13}$ De acuerdo a las guías publicadas en 2016 por la Sociedad Americana de Anestesia Regional y Medicina del Dolor, el uso del ultrasonido (USG) reduce significativamente el riesgo de lesión nerviosa, la incidencia de paresias y con la aplicación óptima de anestésico disminuye la posibilidad de toxicidad sistémica de anestésico local (AL) así como la incidencia de paresias hemidiafragmáticas en miembro superior. ${ }^{14}$ Chan fue el primer anestesiólogo en publicar el uso del USG para visualización directa de las raíces nerviosas del plexo braquial en el surco interescalénico a nivel del cricoides. ${ }^{15}$ La técnica correcta consiste en la colocación de la sonda del USG lineal en posición transversal en el cuello de 3 a $4 \mathrm{~cm}$ superior a la clavícula sobre la vena yugular externa, el objetivo es realizar una hidrodisección con AL que se extienda alrededor de los troncos superior y medio del plexo braquial entre los músculos escaleno anterior y medio (Figura 2). Con el uso del USG la aguja se inserta aplicando una técnica en plano (alineación con el eje largo de la sonda) o una técnica fuera de plano (alienación con el eje corto de la sonda), la primera permite mejor visualización de la aguja, mientras que la segunda proporciona una trayectoria más corta hacia el objetivo (Figura 3). Existen múltiples complicaciones reportadas entre las que destacan: bloqueo del nervio frénico que resulta en parálisis hemidiafragmática, ${ }^{16}$ síndrome de Horner, ${ }^{17}$ bloqueo del nervio laríngeo, ${ }^{18}$ bloqueo epidural alto ${ }^{19}$ o espinal total, ${ }^{20}$ neumotórax,${ }^{21}$ inyección intravascular, ${ }^{22}$ daño nervioso directo, ${ }^{23}$ toxicidad por anestésico local, ${ }^{24}$ mismas que han disminuido de manera considerable como consecuencia de la incorporación del USG. ${ }^{25}$ 


\section{Bloqueo supraescapular y axilar (circunflejo) combinado}

El bloqueo del nervio supraescapular combinado con un bloqueo del nervio axilar proporciona una alternativa eficaz al bloqueo interescalénico en cirugía de hombro, al bloquear estos nervios por separado se generan menos complicaciones y efectos secundarios. ${ }^{26,27}$ Puede utilizarse en pacientes con contraindicaciones de bloqueo interescalénico, un ejemplo son los pacientes con enfermedad pulmonar obstructiva crónica, obesos o con bloqueo interescalénico fallido. El nervio supraescapular está formado por las raíces del plexo braquial C5 y C6, cruza el triángulo posterior profundo del cuello por debajo del músculo omohioideo y el trapecio hasta alcanzar la escápula; donde pasa por la escotadura supraescapular y por debajo del ligamento transverso superior llegando a la fosa infraespinosa. ${ }^{28}$ El nervio supraescapular tiene fibras motoras, sensitivas y simpáticas. Las fibras sensitivas recogen la sensibilidad de $70 \%$ de la articulación del hombro (región posterior, superior de la articulación y cápsula del hombro), bursa subacromial, ligamentos de las articulaciones glenohumeral y acromioclavicular, periostio y tendones de la escápula, pero no inerva las regiones anterior e inferior del hombro ni la piel. Las fibras motoras inervan los músculos supraespinoso e infraespinoso y parte del redondo menor. ${ }^{29} \mathrm{El}$ estudio de Schneider y Connell demostró que en la «técnica a ciegas» mediante la utilización de tomografía axial computarizada es poco probable que la punta de la aguja se dirija hacia nuestro objetivo (escotadura escapular) y en numerosas ocasiones habría que redirigirla, la ventaja de la

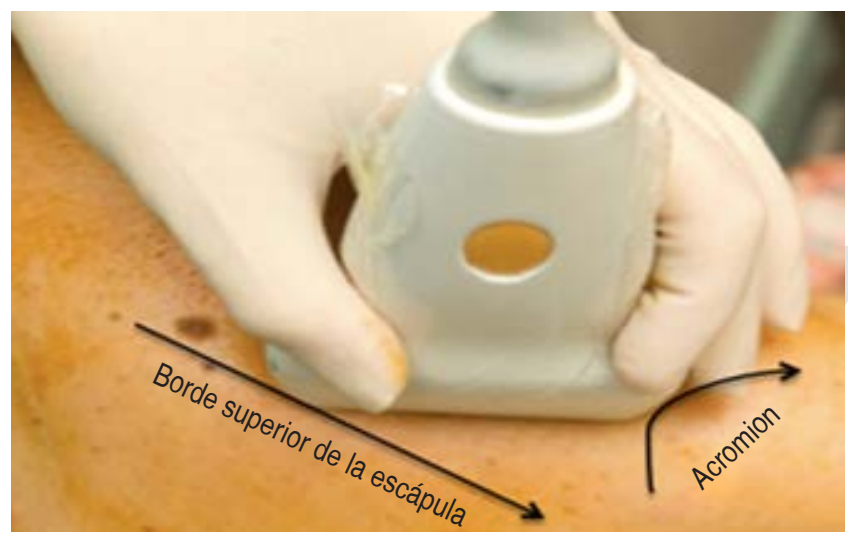

Figura 4: Paciente en posición sedente con los brazos recargados en los muslos, identificaremos la espina de la escápula, el acromion y colocamos la sonda paralela a ésta.

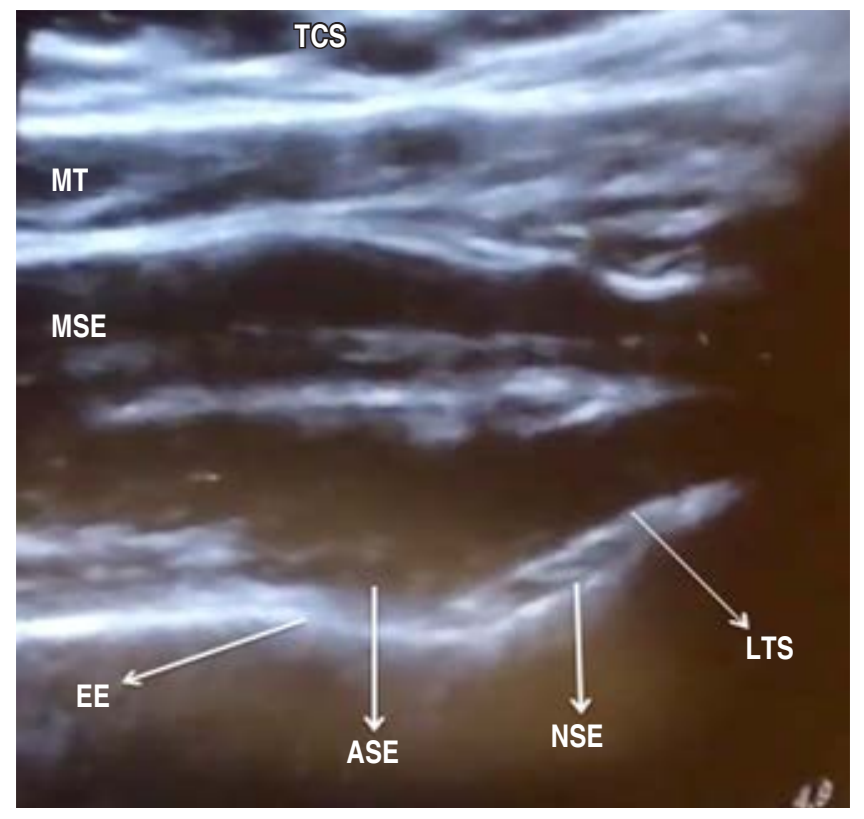

Figura 5: Identificamos tejido celular subcutáneo, músculo trapecio, músculo supraespinoso, arteria supraescapular, escotadura de la escápula, ligamento transverso superior, nervio supraescapular y arteria supraescapular. TCS = tejido celular subcutáneo. MT = músculo trapecio. MSE = músculo supraespinoso. $\mathrm{EE}=$ escotadura de la escápula. $\mathrm{ASE}=$ arteria supraescapular. NSE = nervio supraescapular. LTS = ligamento transverso superior.

aplicación de la técnica con USG es la escasa probabilidad de realizar un neumotórax, ya que bajo visión directa localizamos la escotadura escapular. ${ }^{30}$ La técnica descrita por Harmon y Hearty requiere al paciente en posición sedente con los brazos recargados en los muslos, identificando la espina de la escápula, el acromion y se coloca la sonda paralela a ésta ${ }^{31}(F i$ gura 4). Además se identifica en orden de superficial a profundo: tejido celular subcutáneo, músculo trapecio, músculo supraespinoso, arteria supraescapular, ligamento superior trasverso, nervio supraescapular y arteria supraescapular (Figura 5). La identificación de la escotadura escapular y del ligamento transverso es sencilla; sin embargo, identificar el nervio puede ser complicado, ya que se encuentra en borde lateral de la escotadura. ${ }^{32,33}$ Es importante recordar que las estructuras vasculares (arteria y vena supraescapulares) se localizan encima del ligamento, mientras que el nervio está por debajo, dato que es importante para evitar las inyecciones intravasculares.

El nervio axilar emite ramos para el músculo deltoides y el músculo redondo menor y recoge sensibilidad de la cara superior lateral del brazo, asimismo aporta inervación sensitiva de los $2 / 3$ posteroinferiores del músculo deltoides. ${ }^{7}$ Para el abordaje 
del nervio axilar la sonda suele colocarse paralela al eje longitudinal del húmero aproximadamente 2 $\mathrm{cm}$ por debajo de la porción posterolateral del acromion en la cara posterior del brazo; identificaremos el cuello del húmero, arteria cincunfleja posterior, este nervio se localiza cranealmente en la arteria en el espacio neurovascular entre el músculo redondo menor, deltoides y tríceps. Se introduce la aguja en plano colocando la punta encima de la arteria humeral circunfleja posterior por debajo de la fascia del músculo redondo menor. ${ }^{34}$

\section{Bloqueo de la fascia del erector de la espinal (ESP)}

Los bloqueos interfasciales con ultrasonido se han desarrollado recientemente y representan una nueva vía de aplicación de anestésicos locales en diversas áreas anatómicas; sin embargo, requieren un profundo conocimiento anatómico y funcional que permita establecer un uso óptimo en cuanto a indicación y resultados.

El uso de los bloqueos interfasciales inició en 2007 con la descripción de McDonnell y Hebbard ${ }^{35,36}$ del bloqueo del plano de la fascia del transverso abdominal (TAP), estos estudios fueron precursores de una serie de publicaciones que incluyen la descripción y uso de nuevos bloqueos interfasciales hasta 2016, cuando Forero publica el bloqueo de la fascia del erector espinal y del cual se han descrito diversas aplicaciones clínicas. Forero en su primer reporte describe con éxito el bloqueo ESP para el tratamiento del dolor neuropático en tórax, ${ }^{37}$ que es una técnica anestésica regional cuyo objetivo es depositar el AL en el plano fascial más profundo del músculo erector de la espina (MES) en la punta del proceso transversal de la vértebra. Su principal mecanismo de acción propuesto es la propagación anterior del AL a través de los tejidos conectivos y ligamentos de los agujeros costotransversos, donde las ramas dorsales y ventrales de las raíces de los nervios espinales se originan, tal afirmación dependerá del nivel de bloqueo de la columna torácica y la posterior expansión craneocaudal ${ }^{38,39} \mathrm{El}$ sitio de inyección es distante a la pleura, vasos sanguíneos mayores y médula espinal, con el uso del USG se realiza fácilmente con mínimas posibilidades de complicaciones y muy pocas contraindicaciones. ${ }^{40}$

En hombro se ha utilizado con éxito en síndrome de dolor regional complejo de extremidad superior y en dolor crónico bilateral del mismo. ${ }^{41,42}$ También existen reportes favorecedores en entornos quirúrgicos; se propone con éxito para amputación inte- rescapulotorácica en sarcoma y desarticulación glenohumeral en fracturas patológicas por carcinoma pulmonar logrando analgesias satisfactorias durante 48 horas en infusión continua. ${ }^{43,44}$ Se han documentado series de casos con gran efecto analgésico para fijaciones abiertas de fracturas proximales de húmero, fracturas glenoideas de la escápula, artroplastias totales y parciales de hombro y artroscopias de hombro. ${ }^{45-49}$

La técnica clásica ${ }^{37}$ del bloqueo ESP se realiza en hombro en posición de decúbito lateral o en posición sedente inclinada hacia adelante en el nivel vertebral objetivo (T2, T3, T4 o T5), se coloca un transductor lineal de alta frecuencia en orientación cefalocaudal sobre la línea media de la espalda para identificar el proceso espino vertebral, manteniendo la sonda orientada en sentido cefalocaudal, se desliza la sonda aproximadamente $3 \mathrm{~cm}$ de manera lateral hacia el lado a bloquear, identificando el proceso transverso (PT) como objetivo. Para confirmar la identificación del PT se desliza la sonda más allá del objetivo (diferenciando entre costilla y el PT), siendo la imagen clara y evidente el PT será más superficial y ancho, mientras que la costilla será profunda, más redonda y delgada. Con el transductor en el PT, se inserta la aguja de bloqueo alineada con el eje largo del haz del USG, avanzada en un ángulo de 30 a 45 grados en dirección caudal a cefálica, el operador puede sentir «clics fasciales» correspondientes a la fascia del trapecio, romboides y MES, con un punto final firme al entrar en contacto con el hueso se administra el AL identificando la hidrodisección que separa el MES del PT (Figura 6 A y B). Hasta el momento los volúmenes recomendados son de 20 a $40 \mathrm{~mL}$, los anestésicos locales más utilizados son la bupivacaína y ropivacaína a concentraciones de $0.25-0.5 \%$.

\section{DISCUSIÓN}

Múltiples revisiones han evidenciado el beneficio elemental de las técnicas de anestesia regional al incorporarse a la anestesia multimodal debido al avance de tecnología y al nuevo desarrollo de técnicas que mejoran significativamente la eficacia y seguridad en el perioperatorio. ${ }^{50} \mathrm{El}$ surgimiento de los bloqueos de las fascias ha generado un paso determinante en la nueva anestesia regional moderna, ya que requiere mayores competencias por parte de los anestesiólogos. ${ }^{51}$

El bloqueo de la fascia del erector espinal, por su localización y distribución, puede ser útil en la analgesia de diversos niveles y tipos de cirugía para 

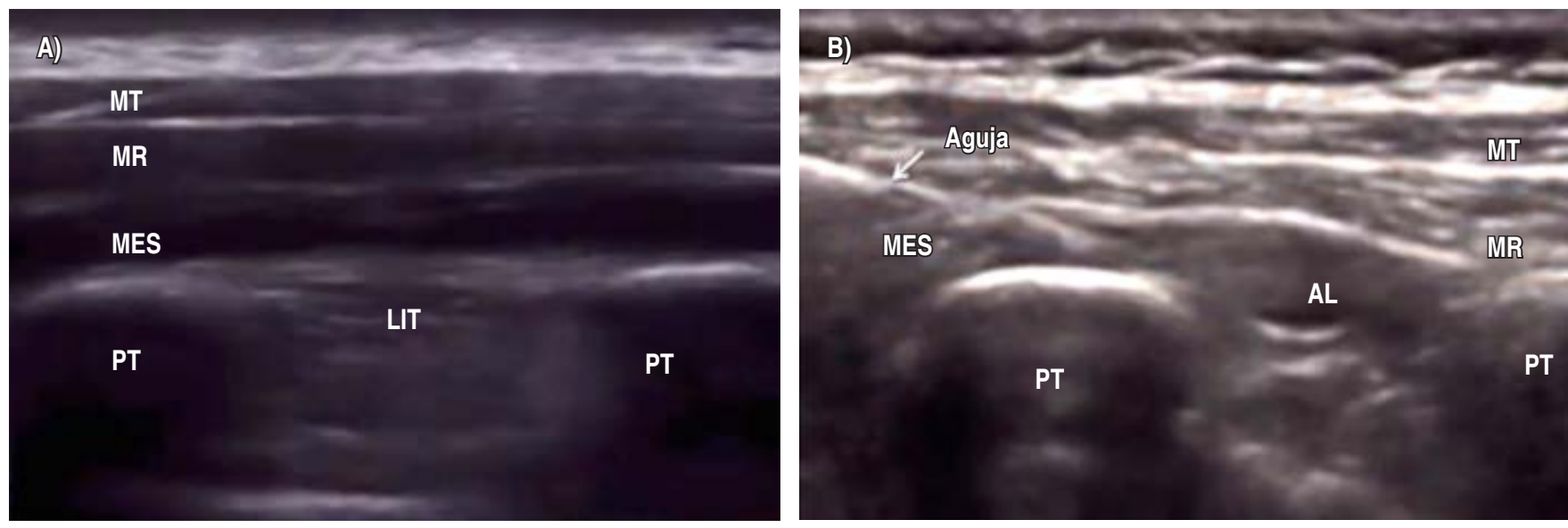

Figura 6: A) Imagen de ultrasonografía a nivel de apófisis transversa de T3, apreciándose músculo trapecio, músculo romboides, músculo erector de la espina, ligamento intertransverso y procesos transversos. B) Imagen en la que se identifica la trayectoria de la aguja disecando fascias musculares y chocando con el PT de T3, se observa zona anecóica por arriba del ligamento intertransverso que representa la hidrodisección con anestésico local. $\mathrm{MT}=$ músculo trapecio. $\mathrm{MR}=$ músculo romboides. $\mathrm{MES}=$ músculo erector de la espina. $\mathrm{LIT}=$ ligamento intertransverso. $\mathrm{PT}=$ procesos transversos. $\mathrm{AL}=$ anestésico local.

manejo de dolor agudo como en cirugía de mama, ${ }^{52}$ cirugía torácica, ${ }^{53}$ reparación de hernia inguinal, ${ }^{54}$ cirugía de columna lumbosacra, ${ }^{55}$ nefrectomía ${ }^{56}$ y cirugía de hombro; ${ }^{45}$ sin embargo, es un procedimiento relativamente nuevo y que está por definir los procedimientos en los que puede ser útil. Aún quedan por definir, analizar y estudiar aspectos relacionados al funcionamiento dinámico y características de las fascias, tanto generales como individuales, continuidad del sistema fascial y la forma en cómo migra el anestésico, papel de la fascia en la innervación local y regional, características regionales e individuales específicas de las fascias.

La anestesia regional se ha estigmatizado como una opción que disminuye los requerimientos de opioides y como una alternativa a la anestesia general, actualmente vale la pena reevaluar este concepto y considerar esta técnica como un complemento o como parte de una estrategia anestésica multimodal que va de la mano con el uso juicioso de opioides, pilares en la anestesia y el tratamiento del dolor agudo. ${ }^{57}$ De acuerdo a las características de dolor postoperatorio que se presentan en cirugías de hombro debemos generar estrategias analgésicas tempranas y agresivas que incorporen la amplia gama de bloqueos regionales guiados por USG existentes para hombro, individualizando a cada uno de los pacientes y adaptándolos a los requerimientos de cada tipo de cirugía.

El campo de investigación y utilidad clínica en la anestesia regional de los bloqueos interfasciales en la actualidad es muy amplio, lo cual de alguna manera nos permitirá establecer con mayor seguridad las aplicaciones clínicas prácticas que tengan como sustento el conocimiento basado en la evidencia científica.

\section{CONCLUSIONES}

El control y resolución de la analgesia en el perioperatorio de los pacientes sometidos a cirugía de hombro son en la actualidad de gran importancia desde el punto de vista económico, social y psicológico; la anestesia regional, y específicamente el bloqueo de la fascia del erector espinal, representa una posibilidad muy importante con resultados que incluyen mayor satisfacción del paciente, menor tiempo de hospitalización, mejores condiciones para la rehabilitación postoperatoria y disminución de complicaciones.

\section{BIBLIOGRAFÍA}

1. Iqbal S, Jacobs U, Akhtar A, Macfarlane RJ, Waseem M. A history of shoulder surgery. The Open Orthop J. 2013; 7: 305309.

2. Andren L, Lundberg BJ. Treatment of rigid shoulders by joint distension during arthrography. Acta Orthop Scand. 1965; 36: 45-53.

3. Beecroft CL, Coventry DM. Anaesthesia for shoulder surgery. Br J Anaesth. 2008; 6: 193-198.

4. Hadzic A, Williams BA, Karaca PE, Hobeika P, Unis G, Dermksian J et al. For outpatient rotator cuff surgery, nerve block anesthesia provides superior same-day recovery over general anesthesia. Anesthesiology. 2005; 102: 1001-1007.

5. Hutton M, Brull R, Macfarlane A. Regional anaesthesia and outcomes. Br J Anaesth. 2018; 18: 52-56. 
An Med (Mex) 2020; 65 (2): 114-121

6. Brow A, Weiss R, Greenberg C, Flatow E, Bigliani L. Interscalene block for shoulder arthroscopy: comparison with general anesthesia. Arthroscopy. 1993; 9: 295-300.

7. Hansen JT. Netter's clinical anatomy. $4^{\text {th }}$. Philadelphia: Elsevier; 2019.

8. Boezart A. Anesthesia and orthopaedic surgery. New York, USA: McGraw Hill; 2006.

9. Hadzic A. Tratado de anestesia regional y manejo del dolor agudo. Hadzic A, editor.: McGrawHill; 2010.

10. Bowns CJ, Sripada R. Regional blockade of the shoulder: approaches and outcomes. Anesthesiol Res Pact. 2012; 2012: 971963.

11. Winnie A. The interescalene brachial plexus black. Anesth Analg. 1970; 49: 455-466.

12. Thomas L, Graham S, Osteen K, Porter H, Nossaman B. Comparison of ultrasound and nerve stimulation techniques for interscalene brachial plexus block for shoulder surgery in a residency training environment: a randomized, controlled, observer-blinded tria. Ochsner J. 2011; 11: 246-252.

13. Vorobeichik L, Brull R, Bowry R, Laffey J, Abdallah F. Should continuous rather than single-injection interscalene block be routinely offered for major shoulder surgery? A meta-analysis of the analgesic and side-effects profiles. Br J Anaesth. 2018; 120: 679-692.

14. Neal J, Brull R, Horn J, Lui S, McCartney C, Perlas A et al. The Second America Society of Regional Anesthesia and pain medicine evidence-based medicine assessment of ultrasoundguied regional anesthesia: executive summary. Reg Anesth Pain Med. 2016; 42: 181-194.

15. Chan V. Applying ultrasound imaging to interscalene brachial plexus block. Reg Anesth Pain Med. 2003; 28: 340-343.

16. El-Boghdadly K, Chin K, Chan V. Phrenic nerve palsy and regional anesthesia for shoulder surgery: anatomical, physiologic, and clinical considerations. Anesthesiology. 2017; 127: $173-191$

17. Stasiowski M, Zuber M, Marciniak R, Kolny M, Chabierska $\mathrm{E}$, Jalowiecki $\mathrm{P}$ et al. Risk factors for the development of Horner's syndrome following interscalene brachial plexus block using ropivacaine for shoulder arthroscopy: a randomised trial. Anaesthesiology Intensive Ther. 2018; 50: 215-220.

18. Thukral S, Gupta P, Gupta M, Lakra A. Recurrent laryngeal nerve palsy following interscalene brachial plexus block: How to manage and avoid permanent sequelae? J Anaesthesiol Clin Pharmacol. 2015; 31: 282-283.

19. Gomez R, Mendes T. Epidural anaesthesia as a complication of attempted brachial plexus blockade using the posterior approach. Anaesthesia. 2006; 6: 591-592.

20. Turner F, Shih R, Fishman I, Calello D, Solano J. Total spinal anesthesia following an interscalene block treated with intravenous lipid emulsion. Cureus. 2019; 11: 4491.

21. Leander-Olsson O, Borglund-Hemph A, Jakobsson J. Pneumothorax following shoulder arthroscopy under combined regional and general anaesthesia-A case report. Int J Surg Case Rep. 2016; 24: 73-76.

22. Loubert C, Williams S, Helie F, Arcand G. Complication during ultrasound-guided regional block: accidental intravascular injection of local anesthetic. Anesthesiology. 2008; 108: 759-760.

23. Yim G, Lin Z, Shirley C, Isherwood P, Power D. The late diagnosis of nerve injuries following interscalene block and shoulder surgery. J Musculoskelet Surg Res. 2019; 3: 141-145.

24. Vasques F, Behr A, Weinberg G, Ori C, Di Gregorio G. A review of local anesthetic systemic toxicity cases since publication of the American Society of Regional Anesthesia recommendations: to whom it may concern. Reg Anesth Pain Med. 2015; 40: 698-705.

25. Lenters T, Davies J, Matsen F. The types and severity of complications associated with interscalene brachial plexus block anesthesia: local and national evidence. J Shoulder Elbow Surg. 2007; 16: 379-387.

26. Price D. The shoulder block: a new alternative to interscalene brachial plexus blockade for the control of posoperative shoulder pain. Anaesthe and Intensive Care. 2007; 35: 575-581.

27. Checcucci G, Allegra A, Bigazzi P, Gianesello L, Ceruso M, Gritti G. A new technique for regional anesthesia for arthroscopic shoulder surgery based on a suprascapular nerve block and an axillary nerve block: an evaluation of the first results. 2008; 24: 689-696.

28. Standring S. Gray's anatomy. 41st ed.: Elsevier; 2016.

29. Fernandes M, Barbosa M, Sousa A, Ramos G. Suprascapular nerve block: important procedure in clinical practice. Rev Bras Anestesiol. 2012; 62: 96-104.

30. Schneider-Kolsky M, Pike J, Connell D. CT-guided suprascapular nerve blocks: a pilot study. Skeletal Radiol. 2004; 33: 277-282.

31. Harmon D, Hearty C. Ultrasound-guided suprascapular nerve block technique. Pain Physician. 2007; 10: 743-746.

32. Yucesoy C, Akkaya T, Ozel O, Cömet A, Tüccar E et al. Ultrasonographic evaluation and morphometric measurements of the suprascapular notch. Surg Radiol Anat. 2009; 31: 409-414.

33. Dangoisse M, Wilson DJ, Glynn CJ. MRI and clinical study of an easy and safe technique of suprascapular nerve blockade. MRI and clinical study of an easy and safe technique of suprascapular nerve blockade. Acta Anaesthesiol Belg. 1994; 45 (2): $49-54$

34. Uz A, Apaydin N, Bozkurt M, Elhan A. The anatomic branch pattern of the axillary nerve. J Shoulder Elbow Surg. 2007; 16: $240-244$.

35. McDonnell J, O’Donnell B, Curley G, Heffernan A, Power C, Laffey J. The analgesic efficacy of transversus abdominis plane block after abdominal surgery: a prospective randomized controlled trial. Anesth Analg. 2007; 104: 193-197.

36. Hebbard P, Fujiwara Y, Shibata Y, Royse C. Ultrasound-guided transversus abdominis plane (TAP) block. Anaesth Intensive Care. 2007; 35: 616-617.

37. Forero M, Adhikary S, Lopez H, Tsui C, Chin K. The erector spinae plane block: a novel analgesic technique in thoracic neuropathic pain. Reg Anesth Pain Med. 2016; 41: 621-627.

38. Yang H, Choi Y, Kwon H, O J, Cho T, Kim S. Comparison of injectate spread and nerve involvement between retrolaminar and erector spinae plane blocks in the thoracic region: a cadaveric study. Anaesthesia. 2018; 73: 1244-1250.

39. Adhikary S, Bernard S, Lopez H, Chin K. Erector spinae plane block versus retrolaminar block: a magnetic resonance imaging and anatomical study. Reg Anesth Pain Med. 2018; 43: 756-762.

40. Luftig J, Mantuani D, Herring A, Dixon B, Clattenburg E, Nagdev A. Successful emergency pain control for posterior rib fractures with ultrasound-guided erector spinae plane block. Am J Emerg Med. 2018; 36: 1391-1396.

41. Bang S, Choi J, Kim E. A high thoracic erector spinae plane block used for sympathetic block in patients with upper extremity complex regional pain syndrome. J Clin Anesth. 2020; 60: 99-100.

42. Forero M, Rajarathinam M, Adhikary S, Chi. Erector spinae plane block for the management of chronic shoulder pain: a case report. Can J Anaesth. 2018; 65: 288-293. 
43. Tsui B, Mohler D, Caruso T, Horn J. Cervical erector spinae plane block catheter using a thoracic approach: an alternative to brachial plexus blockade for forequarter amputation. Can J of Anaesth. 2019; 66: 119-120.

44. Hamadnalla H, Elsharkawy H, Shimada T, Maheshwari K, Esa W, Tsui B. Cervical erector spinae plane block catheter for shoulder disarticulation surgery. Can J Anaesth. 2019; 66: 1129-1131.

45. Diwan S, Nair A. Erector spinae plane block for proximal shoulder surgery: a phrenic nerve sparing block! Turk J Anaesthesiol Reanim. 2019; 1-3.

46. Kilicaslan A, Hacibeyoglu G, Goger E, Uzun S, Ozer M. Combined erector spinae plane and interscalene brachial plexus block for surgical anesthesia of scapula fracture. J Clin Anesth. 2019; 54: 166-167.

47. Ma W, Sun L, Ngai L, Costouros J, Steffner R, Boublik J et al. Motor-sparing high-thoracic erector spinae plane block for proximal humerus surgery and total shoulder arthroplasty surgery: clinical evidence for differential peripheral nerve block? Can J Anaesth. 2019; 66: 1274-1275.

48. Tulgar S, Selvi O, Senturk O, Serifsoy T, Thomas D. Ultrasound-guided erector spinae plane block: indications, complications, and effects on acute and chronic pain based on a single-center experience. Cureus. 2019; 11: e3815.

49. Tsui B, Ip V. Can electrical nerve stimulation guidance assist in cervical erector spinae plane block catheter placement for total shoulder arthroplasty? Can J Anaesth. 2019; 66: 14171418.
50. Barrington M, Uda Y. Did ultrasound fulfill the promise of safety in regional anesthesia? Curr Opin Anaesthesiol. 2018; 31: 649-655.

51. Wahal C, Kumar A, Pyati S. Advances in regional anaesthesia: A review of current practice, newer techniques and outcomes. Indian J Anaesth. 2018; 62: 94-102.

52. Veiga M, Costa D, Brazao I. Erector spinae plane block for radical mastectomy: A new indication? Resv Esp Anestesiol Reanim. 2018; 65: 112-115.

53. Gaballah K, Soltan W, Bahgat N. Ultrasound-guided serratus plane block versus erector spinae block for postoperative analgesia after video-assisted thoracoscopy: a pilot randomized controlled trial. J Cardiothorac Vasc Anesth. 2019; 33: 19461953.

54. Tulgar S, Selvi O, Kapakli M. Erector spinae plane block for different laparoscopic abdominal surgeries: case series. Case Rep Anesthesiol. 2018; 18: 1-3.

55. Melvin J, Schrot R, Chu G, Chin K. Low thoracic erector spinae plane block for perioperative analgesia in lumbosacral spine surgery: a case series. Can J Anaesth. 2018; 65: 10571065.

56. Santonastaso D, de Chiara A, Musetti G, Bagaphou C, Gamberini E, Agnoletti V. Ultrasound guided erector spinae plane block for open partial nephrectomy: only an alternative? J Clin Anesth. 2019; 56: 55-56.

57. Albrecht E, Chin KJ. Advances in regional anaesthesia and acute pain management: a narrative review. Anaesthesia. 2020; 75: 101-110. 